\title{
Editorial
}

\section{Who should take aspirin for primary prophylaxis of coronary heart disease?}

Aspirin is widely used for its analgesic and antiinflammatory properties, and increasingly in recent years as antithrombotic treatment. Daily doses of $75-150 \mathrm{mg}$ effectively inhibit the ability of blood platelets to synthesise thromboxane $\mathrm{A}_{2}$ and stable prostaglandins during their lifespan in the circulation (7-10 days), resulting in inhibition of platelet function ex vivo (impaired platelet aggregation) and in vivo (prolonged skin bleeding time). Because platelets play an important role in thrombosis, aspirin has an antithrombotic effect.

Systematic reviews of randomised controlled trials of antiplatelet drugs (usually aspirin) have shown clinically worthwhile reductions in cardiovascular events (non-fatal myocardial infarction, stroke, and cardiovascular death) when these agents are used in the treatment of patients with acute ischaemia (myocardial infarction, unstable angina, stroke), when used as secondary prophylaxis in patients with chronic ischaemia (previous myocardial infarction, stroke or transient cerebral ischaemic attacks; stable angina; peripheral arterial disease), and when used as prophylaxis in patients with atrial fibrillation. ${ }^{12}$ Antiplatelet treatment (usually with aspirin) is therefore recommended as prophylaxis of cardiovascular disease in these patient groups in national, evidence based guidelines in Scotland ${ }^{3}$ and the rest of the UK. ${ }^{4}$ However, as with all effective drugs, aspirin has adverse effects.

\section{Adverse effects of aspirin}

True aspirin allergy is rare. As with other non-steroidal anti-inflammatory drugs, inhibition by aspirin of gastrointestinal prostaglandin synthesis commonly causes upper gastrointestinal symptoms including dyspepsia, nausea, and vomiting, peptic ulceration, and bleeding. The risk of a major gastrointestinal bleed is increased by about 1 in 250 patient years, even with low dose or modified release aspirin. ${ }^{5}$ Inhibition of renal prostaglandin synthesis may also impair renal function, especially in patients with renal artery stenosis and those receiving angiotensin converting enzyme inhibitor treatment. The antiplatelet effect of aspirin impairs primary haemostasis, resulting in increased risk not only of gastrointestinal bleeding but also of bruising and nosebleeds, postoperative bleeding, and haemorrhagic stroke (the risk of which is increased by about 1 in 2500 patient years ${ }^{6}$.

While these adverse effects of aspirin are generally outweighed by its antithrombotic benefits in secondary prophylaxis of cardiovascular disease, the balance of risks and benefits must be carefully judged when aspirin is prescribed (or taken as self medication) for primary prophylaxis of cardiovascular disease. Because of their lower baseline thrombotic risk, such patients will experience less absolute benefit from aspirin than patients with evidence of cardiovascular disease, for a similar level of relative risk reduction. At the same time the adverse effects of aspirin appear unrelated to thrombotic risk, hence such patients experience a lower ratio of antithrombotic benefit to risk of adverse effects (including bleeding) compared to patients with cardiovascular disease.

\section{Long term aspirin in primary prevention of coronary heart disease: what risk threshold merits treatment?}

In this issue, Sanmuganathan and colleagues ${ }^{7}$ report a systematic review of the benefit and harm from aspirin treatment as long term primary prophylaxis, to determine the cardiovascular and coronary risk thresholds at which benefit in primary prevention of myocardial infarction exceeds harm from serious bleeding. They also sought to determine the absolute benefit, expressed as number needed to treat, to prevent myocardial infarction, net of bleeding complications, at different levels of coronary risk. They identified four randomised controlled trials for meta-analysis. The coronary heart disease event risk in the control groups varied from $0.36-1.33 \%$, while the cardiovascular event risk ranged from $0.67-1.17 \%$. There was no heterogeneity between trials for the end points of cardiovascular events, stroke or all cause mortality. There was significant heterogeneity for myocardial infarction $(p=0.03)$, which the authors postulated might be caused by differences in study populations or design, or might be a chance observation. The analysis was continued despite this heterogeneity. It showed significant reductions overall in all cardiovascular events (by 15\%) and in myocardial infarction (by $30 \%$ ), a non-significant reduction in all cause mortality (by $6 \%$ ), and a non-significant increase in stroke incidence (by $6 \%)$. These results are similar to those in another recent analysis. ${ }^{8}$ The rate of bleeding varied widely in control groups (because of varying definition); however, there was no significant heterogeneity between trials, and overall there was a significant increase in bleeding risk (by 69\%).

Relating absolute benefit (cardiovascular event reduction) to absolute risk (increased bleeding), the authors ${ }^{7}$ observed that at coronary event risks of $1.5 \%$ per year, the five year number needed to treat was 44 to prevent a myocardial infarction, and 77 to prevent a myocardial infarction net of any important bleeding complication. They suggest that at lower coronary event rates, aspirin is not worthwhile (or sometimes harmful in that bleeding risk exceeds antithrombotic benefit).

Sanmuganathan and colleagues are to be congratulated on this analysis. It is hard to argue with their conclusion that advice on aspirin for primary prophylaxis of cardiovascular disease requires formal estimation of absolute coronary event risk; this is now accepted as best practice for all methods of prophylaxis, including lifestyle advice and prescription of drugs for reducing smoking, blood pressure, and blood cholesterol. ${ }^{4}$ Their recommendation is consistent with that of current national guidelines within the UK. In Scotland, the SIGN (Scottish Intercollegiate Guideline Network) guideline recommends that "aspirin ( $75 \mathrm{mg} /$ day) should be considered for primary prophylaxis of myocardial infarction in men and women at high risk (for example, $2 \%$ per year) balanced against the 
increased risk of bleeding". ${ }^{3}$ The guideline for the rest of the $\mathrm{UK}^{4}$ is more vague: "aspirin $(75 \mathrm{mg})$ is recommended in individuals who are older than 50 years and are either well controlled hypertensive patients or men at high risk of CHD [coronary heart disease]".

As might be expected, the authors, who have previously advocated a "Sheffield table" to identify a 3\% annual risk of CHD for consideration of statin prescription, advocate another "Sheffield table" to identify a $1.5 \%$ annual risk of $\mathrm{CHD}$ for aspirin prescription. The relative merits of different CHD risk prediction equations or tables remains a controversial topic. I suspect that many doctors and nurses will prefer systems which, rather that predicting "cut-offs", predict the absolute risk of coronary and/or cardiovascular events in an individual patient, then use this as a basis to discuss an individual approach to primary prophylaxis, considering the whole range of effective interventions including aspirin, as well as the individual's risks of adverse effects and their preferences, after informed discussion.

In addition, doctors should advise their patients who take regular aspirin to reduce their risk of cardiovascular events, but those whose risk of CHD is less than $1 \%$ per year should be advised that the adverse effects of aspirin may outweigh its benefits.

\section{What patient characteristics, apart from absolute CHD risk, should influence prescription or advice on aspirin?}

Subgroup analysis in one of the four trials of aspirin in primary prophylaxis (in US physicians) raised the possibility that aspirin may have been more effective in those aged 50 years or more, and when cholesterol concentrations were low rather than high. ${ }^{9}$ Subgroup analysis in another of the four trials (in UK general practice) did not confirm this hypothesis, but raised the possibility that aspirin may have been more effective in those with low rather than high blood pressure. ${ }^{10}$ These subgroup analyses should be viewed with caution, because they are retrospective, based on small numbers of events, hypothesis generating, and await hypothesis testing in future systematic reviews, including data from studies in progress. ${ }^{8}$ Meta-analysis of secondary prevention trials of aspirin and other antiplatelet agents has not shown significant differences in subgroups. ${ }^{1}$

In addition to standard contraindications, caution is required when considering prescription of aspirin to patients at increased risk of gastrointestinal bleeding (for example, active dyspepsia, previous peptic ulcer without eradication of Helicobacter pylori) or intracranial bleeding (for example, uncontrolled hypertension ${ }^{6}$ ). Many patients at increased risk of CHD are hypertensive, and it is sensible to control this before regular aspirin use. ${ }^{4}$

One subgroup who may benefit particularly from primary aspirin prophylaxis are those with asymptomatic evidence of atherosclerosis. In the ongoing AAA (aspirin in asymptomatic atherosclerosis) trial, men and women aged 50-79 years who have no clinical evidence of cardiovascular disease, but have an ankle-brachial systolic blood pressure index less than 0.95 (which is associated with increased cardiovascular risk, independent of classical risk factors ${ }^{11}$ ), are randomised to low dose aspirin or placebo. The results are expected in 2005 .

University of Glasgow,

GORDON D O LOWE

Department of Medicine,

Royal Infirmary,

Glasgow, G31 2ER, UK

gdl1j@clinmed.gla.ac.uk

The author has received departmental support from the British Heart Foundation and the Chief Scientist Office, The Scottish Office for trials of aspirin (including primary prevention), and from the following pharmaceutical companies for trials of aspirin and/or other antithrombotic agents: AstraZeneca, Boehnies for trials of aspirin and/or other antithrombotic agents: AstraZeneca, Boeh-
ringer Ingelheim, Bristol-Myers-Squibb, Glaxo, Knoll, Leo, Rhone-Poulencringer Ingelheim, Bristol-Myers-Squibb, Glaxo, Knoll, Leo, Rhone-Poulenc-
Rorer, Sanofi-Winthrop, SmithKline Beecham. The author has also received honoraria and travel expenses for lectures and occasional consultancies with some of these bodies and from: Boehringer Mannheim, Eisai, Mitsubishi Kasei, and NovoNordisk.

1 Antiplatelet Trialists' Collaboration. Collaborative overview of randomised trials of antiplatelet therapy. I. Prevention of death, myocardial infarction and stroke by prolonged antiplatelet therapy in various categories of patients. BMF 1994;308:81-106 (and update at 1997 Antithrombotic Trialists' Collaboration meeting)

2 Chen Z, Sandercock P, Pan H, et al. Indications for early aspirin use in acute ischaemic stroke. A combined analysis of 40,000 randomized patients from the Chinese acute stroke trial and the international stroke trial. Stroke 2000;31:1240-9.

3 Scottish Intercollegiate Guideline Network (SIGN). Antithrombotic therapy. A national clinical guideline. Edinburgh: SIGN, 1999.

4 Wood D, Durrington P, Poulter N, et al. Joint British recommendations on prevention of coronary heart disease in clinical practice. Heart 1998; 80(suppl 2): $\mathrm{S} 1-29$.

5 Derry S, Loke YK. Risk of gastrointestinal haemorrhage with long term use of aspirin: meta-analysis. BMF 2000;321:1183-7.

$6 \mathrm{He} \mathrm{J}$, Whelton PK, Vu B, et al. Aspirin and risk of haemorrhagic stroke. A meta-analysis of randomised controlled trials. $7 A M A$ 1998;280:1930-35.

7 Sanmuganathan PS, Gharamani P, Jackson PR, et al. Aspirin for primary prevention of coronary heart disease: safety and absolute benefit related to coronary risk derived from meta-analysis of randomised trials. Heart 2001; 85:265-71

8 Sudlow C, Baigent C. What is the role of antithrombotic treatment in asymptomatic people? Clinical Evidence 1999;2:60-71

9 Steering Committee of the Physicians Study Research Group. Final report on the aspirin component of the ongoing physicians study. $N$ Engl F Med 1989;321:129-35.

10 Meade TW, Brennan PJ on behalf of the MRC General Practice Research Framework. Determination of who may derive most benefit from aspirin in primary prevention; subgroup results from a randomised controlled trial. BMF 2000;321:13-17.

11 Leng GC, Fowkes FGR, Lee AJ, et al. Use of ankle brachial pressure index to predict cardiovascular events and death: a cohort study. BMF 1996;313: $1440-4$. 